\title{
The JAK inhibitor tofacitinib ameliorates immune-mediated liver injury in mice
}

\author{
HAN WANG, XINXIA FENG, PING HAN, YU LEI, YUJIA XIA, DEAN TIAN and WEI YAN \\ Department of Gastroenterology, Tongji Hospital, Tongji Medical College, \\ Huazhong University of Science and Technology, Wuhan, Hubei 430030, P.R. China
}

Received March 11, 2019; Accepted August 8, 2019

DOI: $10.3892 / \mathrm{mmr} .2019 .10750$

\begin{abstract}
The prevalence of immune-mediated liver diseases such as autoimmune liver disease or viral hepatitis has increased in recent years, and the side effects of pre-existing treatments are a worldwide problem. Regulatory $\mathrm{T}$ cells (Tregs) and T helper 17 (Th17) cells play important roles in the development of immune-mediated hepatitis and may serve as potential therapeutic targets. Tofacitinib, a new Janus kinase (JAK) inhibitor, is under investigation for the treatment of rheumatoid arthritis; it is also helpful in treating ulcerative colitis and psoriasis. The roles of tofacitinib were investigated in conferring protection against immune-mediated liver injury in mice. $\mathrm{T}$ cell-mediated hepatitis was induced by concanavalin A (ConA). The mice in the treatment groups were administered with tofacitinib intragastrically before the ConA injection. Histopathological examination was performed by hematoxylin and eosin (H\&E) staining, and the serum transaminase and inflammatory cytokine levels were determined using an automatic biochemistry analysis apparatus or cytometric bead array (CBA) kits. Flow cytometric analysis was used to detect Tregs and Th17 cells. Tofacitinib significantly decreased the hepatic injury induced by ConA and prominently decreased the liver transaminase level. The secretion of several anti-inflammatory cytokines such as interleukin (IL)-10 was upregulated in mice from the treatment group, compared to that in mice treated with ConA alone, while the expression of interferon- $\gamma($ IFN- $\gamma)$ and tumor necrosis factor- $\alpha$ (TNF- $\alpha$ ) decreased. Tofacitinib treatment increased the number of Tregs and reduced the number of Th17 cells. Furthermore, tofacitinib could relieve liver fibrosis under conditions of autoimmune hepatitis (AIH). The present
\end{abstract}

Correspondence to: Professor Dean Tian or Professor Wei Yan, Department of Gastroenterology, Tongji Hospital, Tongji Medical College, Huazhong University of Science and Technology, 1095 Jiefang Avenue, Wuhan, Hubei 430030, P.R. China

E-mail: datian@tjh.tjmu.edu.cn

E-mail: yanwei@tjh.tjmu.edu.cn

Key words: tofacitinib, concanavalin A, immune-mediated liver injury, autoimmune hepatitis, Treg, Th17 results indicated that tofacitinib improved immune-mediated hepatitis and restored the impaired Treg/Th17 cell ratio, which suggests that it may serve as a novel treatment approach for immune-mediated liver diseases.

\section{Introduction}

Immune-mediated liver injury is one of the most troublesome diseases clinically. Autoreactive T cells destroy hepatocytes or cholangiocytes in autoimmune liver diseases and virus-specific $\mathrm{T}$ cells destroy infected hepatocytes in viral hepatitis (1). The pathogenesis of immune-mediated liver injury has not yet been fully clarified (2), and immunosuppressive treatments or antiviral drugs are the main therapeutic methods used clinically for treating these diseases (3). However, the questionable effectiveness and strong side-effects of these treatments and the high possibility of disease relapse limit the utility of these therapeutic approaches. Hence, finding new treatments to ameliorate immune-mediated liver damage in these patients remains an urgent issue.

An imbalance between $\mathrm{CD} 4{ }^{+} \mathrm{CD} 25^{+} \mathrm{Foxp} 3^{+}$regulatory $\mathrm{T}$ cells (Tregs) and $\mathrm{T}$ helper 17 (Th17) cells has recently been suggested to be important in the pathogenesis of immune-mediated liver diseases (4). Tregs are essential in maintaining peripheral immunological tolerance, as they control autoreactive $\mathrm{T}$ cells and inhibit inflammation by releasing anti-inflammatory cytokines (5). Th17 cells are a subtype of helper $\mathrm{CD}^{+}{ }^{+} \mathrm{T}$ cells that produce interleukin (IL)-17, which induces immune cell infiltration and liver damage, driving hepatic inflammation and contributing to autoimmune liver disease (6,7). Moreover, an increase in the Th17/Treg ratio also accelerates liver fibrosis (8). A recent study has indicated that blocking Th17 cells allows CD25- Treg cells to differentiate into functionally stable immune inhibitory cells; this may be a novel therapy for patients with autoimmune hepatitis (AIH) (7). The developmental pathways for Th17 cells and Tregs are reciprocally interconnected (9), suggesting that the factors that affect the balance between these two cell types may influence the outcome of the immune responses.

Concanavalin A (ConA) can induce a typical $\mathrm{T}$ cell-mediated hepatitis in mice, which is characterized by significantly increased plasma levels of transaminase and severe liver cell inflammation or even necrosis within 8-24 h $(10,11)$. ConA has the ability to stimulate the activation 
of $\mathrm{T}$ lymphocytes, mostly CD4 ${ }^{+} \mathrm{T}$ cells, and animal models of ConA-induced liver injury are ideal tools to investigate T-cell-dependent immune-mediated liver injury (12). The infiltration of $\mathrm{T}$ cells, macrophages, and neutrophils into the mouse liver and the elevated levels of pro-inflammatory cytokines produced from these cells indicate that this mouse model closely mimics the pathogenic mechanisms and pathological changes associated with AIH $(13,14)$. Moreover, interferon- $\gamma($ IFN- $\gamma)$ and tumor necrosis factor- $\alpha$ (TNF- $\alpha)$ are the two most important cytokines mediating ConA-induced immune-mediated hepatitis; they are also the most crucial cytokines associated with autoimmune liver diseases in the human body $(15,16)$. However, the ConA-induced mouse model is an acute immune-mediated mouse model. The liver inflammation and lymphocyte infiltration disappear in $48 \mathrm{~h}$. For decades, researchers have been investigated the chronic immune-mediated mouse model $(17,18)$. Cytochrome P450 2D6 (CYP2D6) is the major human autoantigen in type $2 \mathrm{AIH}$ and recognized by type 1 liver kidney microsomal antibodies (LKM-1s) (19). Overexpression of the human CYP2D6 gene in mice can result in a chronic form of severe, autoimmune liver damage, and autoantibody generation $(20,21)$. Chronic hepatitis and liver fibrosis can also be observed in a CYP2D6 mouse model, suggesting that it could be an appropriate tool to investigate chronic immune-mediated hepatitis.

Members of the Janus kinase (JAK) family, including JAK1, JAK2, JAK3, and tyrosine kinase 2 (Tyk2), are involved in the growth, survival, development, and differentiation of a variety of cells, but are critically important for immune cells (22). JAKs phosphorylate signal transducers and activators of transcription (STATs) to regulate the expression of different downstream genes (23). JAKs are essential for cytokine-induced intracellular signaling of lymphocytes, and their dysfunction contributes to the impairment of immune cell function (24). Cytokines that bind to receptors containing the common $\gamma$ chains, such as IL-2, IL-4, IL-7, IL-9, IL-15, and IL-21, are crucial for the function of T cells; JAK1 is activated via these cytokine-binding chains, while the common $\gamma$ chains activate JAK3 (25). Nowadays, the clinical use of JAK inhibitors has been confirmed to improve many inflammation-driven diseases.

Tofacitinib is a potent, selective JAK inhibitor that preferentially inhibits JAK1 and JAK3 (26). It is a new JAK inhibitor that is under investigation for the treatment of rheumatoid arthritis (27); according to recent studies, it has also been reported to be helpful against psoriatic arthritis (28), ulcerative colitis (29), and alopecia areata (30). Tofacitinib inhibits STAT-1 activation, resulting in the downregulation of IL- 6 and IFN- $\gamma$ in naïve CD4 ${ }^{+} \mathrm{T}$ cells (31). Evidence has revealed that tofacitinib may improve autoimmune diseases by suppressing the differentiation of pathogenic Th1 and Th17 cells, as well as innate immune cell signaling (31). However, the exact immune processes affected by tofacitinib and the influence of tofacitinib on gene expression in situ are unknown (27). Furthermore, there are no studies reporting the effects of tofacitinib on the balance of Tregs and Th17 cells or immune-mediated hepatitis. The present study aimed to investigate the effects of tofacitinib on immune-mediated liver injury in mice and the mechanisms underlying these effects.

\section{Materials and methods}

Reagents. Tofacitinib was purchased from Dalian Meilun Biotechnology Co.,Ltd. ConA and dimethyl sulfoxide (DMSO) were purchased from Sigma-Aldrich; Merck KGaA. The antibodies used for western blotting and immunohistochemistry in this study were purchased from Santa Cruz Biotechnology, Inc., R\&D Systems, and Cell Signaling Technology, Inc., including antibodies against STAT1, phosphorylated STAT1 (p-STAT1), TNF- $\alpha$, and IFN- $\gamma$. The antibodies used for flow cytometry, such as those recognizing CD4, CD25, Foxp3, and IL-17A were purchased from BioLegend, Inc. and BD Pharmingen; BD Biosciences. Fetal bovine serum (FBS) was purchased from Gibco; Thermo Fisher Scientific, Inc.

Plasmid and in vivo gene transfection. Plasmid pCYP2D6, the expression vector carrying the cDNA encoding human CYP2D6, was constructed by the insertion of cDNA into plasmid pcDNA3.1 (Invitrogen; Thermo Fisher Scientific, Inc.) in our laboratory. For in vivo gene transfection, pCYP2D6 was injected to mice via the tail vein using the hydrodynamics-based gene delivery technique (32).

Animals and experimental protocol. Specific pathogen-free (SPF) male C57BL/6 mice (6-8 weeks old; $18-20 \mathrm{~g}$ ) were purchased from Beijing Vital River Laboratory Animal Technology Co., Ltd. The mice were housed in an SPF environment at $24 \pm 2^{\circ} \mathrm{C}$ with an alternating 12 -h light/dark cycle at the Experimental Animal Center of the Tongji Medical College. A total of 120 mice were used in the whole experiment and 6 mice were assigned to each experimental group except when otherwise indicated in the figure legends. Ninety-six of those mice were used to study the effect of tofacitinib in the ConA-induced immune-mediated liver injury and the remaining mice were used to confirm the effect of tofacitinib on liver fibrosis in an AIH mouse model. For all mouse experiments, the method of euthanasia was cervical dislocation. The protocol was approved by the Ethics Committee of Animal Experiments of Tongji Medical College and monitored by the Department of Experimental Animals of Tongji Medical College.

The treatment was as follows for the ConA mouse model: The mice were injected with a single dose $(15 \mathrm{mg} / \mathrm{kg}$ of body weight) of ConA via the tail vein to induce acute immune-mediated liver injury, as described in a previous study (12). Three days before the injection of ConA, the mice in the treatment groups were administered with tofacitinib $(5,10$ and $15 \mathrm{mg} / \mathrm{kg} /$ day $)$ by gavage based on the recommended dose described in a previous study (33). The mice were sacrificed at 12,24 and $48 \mathrm{~h}$ post-ConA injection. The blood was collected from the angular vein and the livers were collected for hematoxylin and eosin (H\&E) staining, immunohistochemistry (IHC), western blot analysis, and quantitative polymerase chain reaction (qPCR). For the AIH mouse model: To detect the effects of tofacitinib on the mouse model of chronic immune-mediated hepatitis, adenovirus $\left(10^{9} \mathrm{pfu}\right.$; Viraltherapy Technology) was injected once initially and then pCYP2D6 plasmid was transfected several times $(50 \mu \mathrm{g}$ per injection) into mice to induce the AIH mouse model, as described in our previous studies $(34,35)$. Thirty-six days after 
the adenovirus injection, the AIH mice were administered with tofacitinib (10 mg/kg/day, 2 days apart) by gavage and were then sacrificed two weeks later to observe the level of liver fibrosis by Sirius red staining.

Histopathology and immunohistochemistry. The entire left lobe of the mouse livers was excised and fixed in $4 \%$ paraformaldehyde for at least $24 \mathrm{~h}$, embedded in paraffin, and cut to yield 5- $\mu \mathrm{m}$-thick sections. Following hydration in a decreasing ethanol gradient, all sections were deparaffinized and stained with Harris hematoxylin solution for $5 \mathrm{~min}$ at $37^{\circ} \mathrm{C}$. For IHC, paraffin-embedded liver tissue samples were cut into $5-\mu \mathrm{m}$-thick consecutive sections, dewaxed in xylene, and rehydrated in graded ethanol solutions. Then, the nonspecific binding sites in the tissues were blocked, and the steam cooking method was used for antigen retrieval. The sections were incubated with the following primary antibodies: Anti-p-STAT1 (1:50; product no. 7649; Cell Signaling Technology, Inc.); anti-IL-6 (1:100; cat. no. GB11117) and anti-IL-10 (1:100; cat. no. GB11108; both from Servicebio Technology Co., Ltd.) overnight at $4^{\circ} \mathrm{C}$. Then, they were incubated with horseradish peroxidase-conjugated polyclonal goat anti-rabbit secondary antibodies (1:200; GB23303; Servicebio Technology Co., Ltd.) for $1 \mathrm{~h}$ at room temperature. Finally, the sections were counterstained with hematoxylin.

Protein extraction and western blot analysis. Cell extracts and liver tissue samples were digested in RIPA buffer containing a phosphatase inhibitor cocktail and PMSF (Wuhan Boster Biological Technology, Ltd.). These samples were centrifuged at $12,000 \mathrm{x} \mathrm{g}$ for $30 \mathrm{~min}$ and the supernatant was retained. The protein concentration was determined using the bicinchoninic acid method. Then, $30 \mu \mathrm{g}$ of each protein sample was separated on $10 \%$ SDS polyacrylamide gels; the protein bands were then transferred onto PVDF membranes. The membranes were blocked with 5\% non-fat milk (non-fat dry milk powder dissolved in Tris-buffered saline with Tween-20) for $1 \mathrm{~h}$ at room temperature and then incubated overnight at $4{ }^{\circ} \mathrm{C}$ on shaking tables with the following primary antibodies: Anti-STAT1 (1:1,000; product no. 14994) and anti-p-STAT1 (1:1,000; product no. 7649; both from Cell Signaling Technology, Inc.); anti-IFN- $\gamma$ (1:2,000; cat. no. AF-485; R\&D Systems); anti-TNF- $\alpha$ (1:500; cat. no. sc-12744; Santa Cruz Biotechnology, Inc.); and anti- $\beta$-actin $(1: 2,000$; Promoter Biotechnology Ltd.). Next, the membranes were washed and incubated with horseradish peroxidase-conjugated secondary antibodies (1:1,000; Promoter Biotechnology Ltd.) for $1 \mathrm{~h}$ at room temperature. $\beta$-actin was used as a loading control. The expression of the antibody-linked proteins was visualized by enhanced chemiluminescence using an ECL assay kit (Wuhan Boster Biological Technology, Ltd.) and analyzed using ImageJ V1.48 (National Institutes of Health).

RNA extraction and real-time qPCR. Total RNA was isolated from the liver tissue samples and cells using TRIzol reagent (Takara Bio, Inc.) and transcribed into cDNA using a reverse transcription kit (cat. no. RR036A; Takara Bio, Inc.), according to the manufacturer's protocols. qPCR was performed according to the following steps: 40 cycles at $95^{\circ} \mathrm{C}$ for $30 \mathrm{sec}$ and $60^{\circ} \mathrm{C}$ for $30 \mathrm{sec}$; the PCR analyses were performed using
Table I. Primer sequences for PCR.

\begin{tabular}{ll}
\hline Genes & \multicolumn{1}{c}{ Primer sequences } \\
\hline IFN- $\gamma$ & F: 5'-TGCTGATGGCCTGATTGTCTT-3' \\
& R: 5'-GCCACGGCACAGTCATTGA-3' \\
TNF- $\alpha$ & F: 5'-CTGAACTTCGGGGTGATCGG-3' \\
& R: 5'-GGCTTGTCACTCGAATTTTGAGA-3' \\
$\beta$-actin & F: 5'-GCTCTTTTCCAGCCTTCCTT-3' \\
& R: 5'-TGATCCACATCTGCTGGAAG-3' \\
\hline
\end{tabular}

IFN- $\gamma$, interferon $\gamma$; TNF- $\alpha$, tumor necrosis factor- $\alpha$; F, forward; $\mathrm{R}$, reverse.

Maxima SYBR-Green qPCR Master Mixes (Takara Bio, Inc.) on an ABI StepOne Real-Time PCR system (Applied Biosystems; Thermo Fisher Scientific, Inc.). Fold significance was determined by the $2^{\Delta \Delta \mathrm{Cq}}$ method, as described in a previous study (36). The primers used for real-time qPCR are listed in Table I.

Assay for serum transaminase activity or inflammatory cytokines. Venous blood was collected from the angular vein of the mice and then centrifuged at $500 \mathrm{x} \mathrm{g}$ for $10 \mathrm{~min}$ to obtain serum as the supernatant. The activities of liver enzymes, including aspartate transaminase (AST) and alanine transaminase (ALT), in the collected mouse sera, were determined using an automatic biochemistry analysis apparatus at the Clinical Laboratory of Tongji Hospital. The cytokines (IL-2, IL-4, IL-6, IFN- $\gamma$, TNF- $\alpha$, IL-17A, and IL-10) in the serum were detected by flow cytometry using a mouse cytometric bead array (CBA) kit (560485; BD Biosciences).

\section{Cell isolation}

Isolation of liver mononuclear cells (MNCs). To isolate the non-parenchymal cells (NPCs) from the liver, first, in situ perfusion with DMEM/F12 (Thermo Fisher Scientific, Inc.) containing type IV collagenase (Sigma-Aldrich; Merck KGaA) was performed. Mice in each experimental group were anesthetized using a mixture of ketamine $(100 \mathrm{mg} / \mathrm{kg})$ and xylazine $(10 \mathrm{mg} / \mathrm{kg})$ by intraperitoneal injection. The liver was then perfused, ground, digested, and filtered. The filtrate was centrifuged at $20 \mathrm{x}$ g for $5 \mathrm{~min}$ and washed in DMEM/F12. The resuspended cells were processed with $30 \%$ Percoll (Sigma-Aldrich; Merck KGaA) and gently overlaid onto $70 \%$ Percoll. After density gradient centrifugation at $1,000 \mathrm{x} \mathrm{g}$ for $30 \mathrm{~min}$, liver MNCs were harvested from the interface of the Percoll gradient (37). The MNCs collected were used for further fluorescence-activated cell sorting (FACS) analysis.

Isolation of splenocytes. Mice spleens were ground and filtered with $70 \mu \mathrm{m}$ cell strainer in PBS. After elimination of erythrocytes by Red Blood Cell Lysis Buffer (BD Biosciences), splenocytes were washed and resuspended in PBS for further FACS analysis.

Flow cytometry. A single-cell suspension of MNCs (at least $10^{6}$ cells/tube) was resuspended in phosphate-buffered 
A
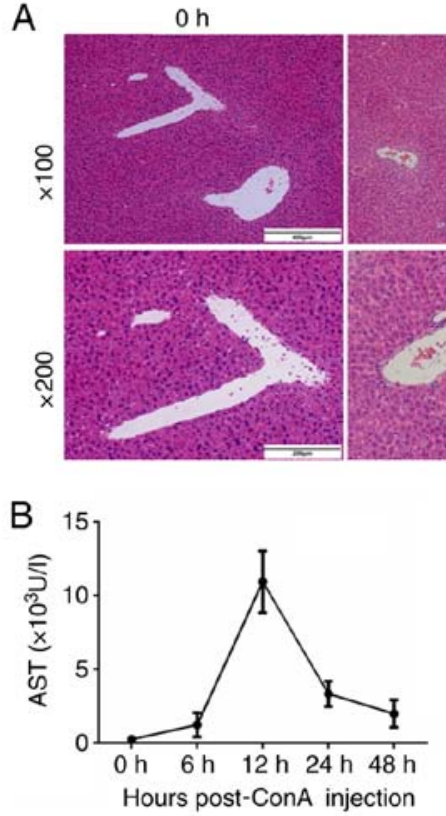

$6 \mathrm{~h}$
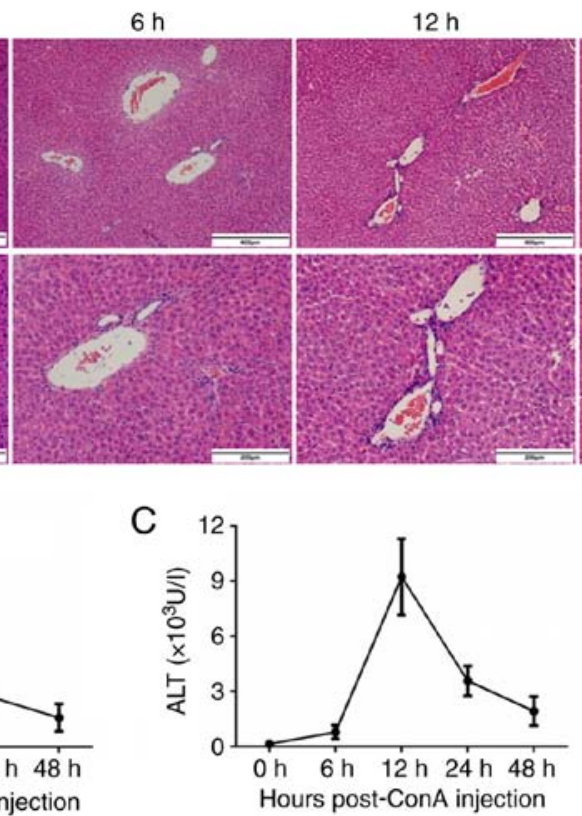
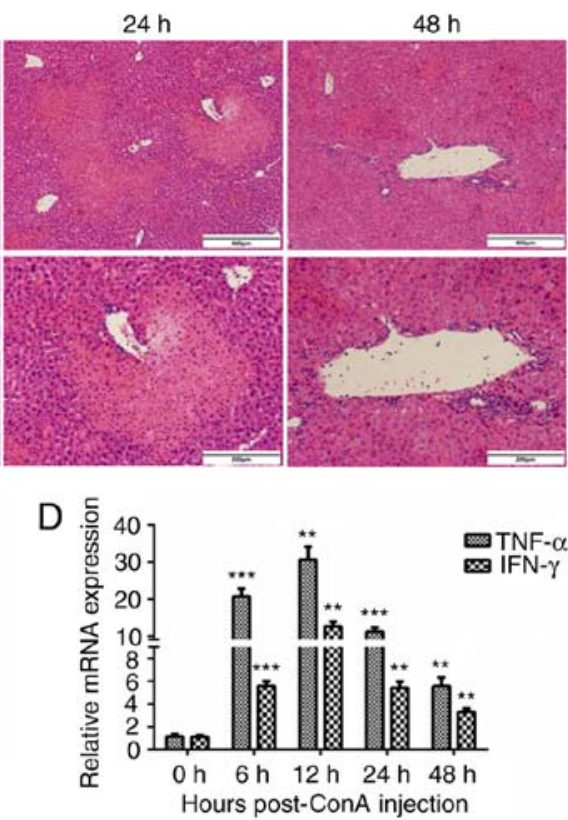

Figure 1. Immune-mediated hepatitis is induced by ConA injection. (A) C57 BL/6 mice were injected with ConA (15 mg/kg) via the tail vein and sacrificed at $0,6,12,24$, and $48 \mathrm{~h}$ post-injection. The liver tissues were harvested and analyzed by H\&E staining ( $\mathrm{n}=6$ in each group; $\mathrm{x} 100$ and $\mathrm{x} 200 \mathrm{magnification})$. (B and C) The plasma ALT and AST levels of the mice were detected using an automatic biochemistry analysis apparatus (n=6). (D) The mRNA expression of TNF- $\alpha$ and IFN- $\gamma$ in the mouse livers was analyzed by real-time PCR $(n=6) .{ }^{* *} \mathrm{P}<0.01,{ }^{* * *} \mathrm{P}<0.001$ vs. the 0 -h group. ConA, concanavalin A; ALT, alanine transaminase; AST, aspartate transaminase; TNF- $\alpha$, tumor necrosis factor- $\alpha$; IFN- $\gamma$, interferon- $\gamma$.

saline (PBS) containing $1 \%$ BSA. To reduce nonspecific fluorescent staining, the cells were incubated with anti-mouse CD16/CD32 (cat. no. 101319; BioLegend, Inc.), which blocked the Fc $\gamma$ III/II receptor. Then, the surfaces of the cells were stained with fluorochrome-conjugated antibodies for $30 \mathrm{~min}$ on ice. The following antibodies were used: FITC-conjugated anti-CD4 (cat. no. 557307), APC-conjugated anti-CD25 (cat. no. 557192), BV421-conjugated anti-Foxp3 (cat. no. 562996), and PE-conjugated anti-IL-17A (cat. no. 559502; all from BD Biosciences). The cell samples were assessed on a FACS Calibur flow cytometer (BD Immunocytometry Systems) and the data were analyzed using the FlowJo software V10 (Tree Star, Inc.).

Statistical analysis. All data are expressed as the mean \pm standard error and all experiments were performed independently in triplicate. One-way analysis of variance (one-way ANOVA) with Tukey's multiple comparisons test or Dunnett's multiple comparisons test if the P-value was significant, were used. Statistical analysis was performed with GraphPad Prism 5.0 (GraphPad Software). P<0.05 was considered to indicate a statistically significant difference.

\section{Results}

The JAK1/STAT1 pathway is activated in mice with ConA-induced hepatitis. To establish a mouse model of immune-mediated liver injury, mice were injected with ConA (15 mg/kg) via the tail vein and sacrificed at $0,6,12,24$, and $48 \mathrm{~h}$ to observe the degree of inflammation in the livers. The liver tissues were harvested, and the levels of inflammation were analyzed by H\&E staining (Fig. 1A). As revealed in the representative images, a handful of inflammatory cells began to infiltrate around the portal area $6 \mathrm{~h}$ post-injection, and more inflammatory cells had accumulated at $12 \mathrm{~h}$ post-injection. In addition, abundant necrotic areas were observed at $24 \mathrm{~h}$ after the ConA injection. The plasma ALT and AST levels increased $6 \mathrm{~h}$ after the intravenous ConA administration and peaked at $12 \mathrm{~h}$ (Fig. $1 \mathrm{~B}$ and C). Real-time PCR was also used to detect the mRNA levels of the important inflammatory cytokines TNF- $\alpha$ and IFN- $\gamma$. Their expression patterns revealed clear tendencies, peaking at $12 \mathrm{~h}$ after the ConA injection (Fig. 1D).

To further explore whether the JAK1/STAT1 pathway is involved in ConA-induced acute immune-mediated liver injury, western blotting and immunohistochemical staining were used to detect the expression of STAT1 and p-STAT1 in the mouse livers at different time-points (Fig. 2A and B). The expression of p-STAT1 increased at the 12-h time-point, and further significantly increased at $24 \mathrm{~h}$. However, the p-STAT1 levels revealed a decreasing trend at the 48-h time point, indicating that the JAK1/STAT1 pathway plays an important role in the progression and development of ConA-induced acute liver injury in mice. These results revealed that ConA could in fact induce acute hepatitis in mice and that the JAK1/STAT1 pathway was activated in this immune-mediated hepatitis mouse model, indicating that this pathway may play a key role in ConA-induced mouse hepatitis.

Tofacitinib effectively ameliorates inflammation in the ConA-induced hepatitis mouse model. To detect the effects of tofacitinib on ConA-induced hepatitis in vivo, the mice were randomly divided into different experimental groups. The mice in the treatment groups received different doses of tofacitinib $(5,10$, and $15 \mathrm{mg} / \mathrm{kg} /$ day) three days before the ConA injection to ascertain whether tofacitinib exerts protective effects and 


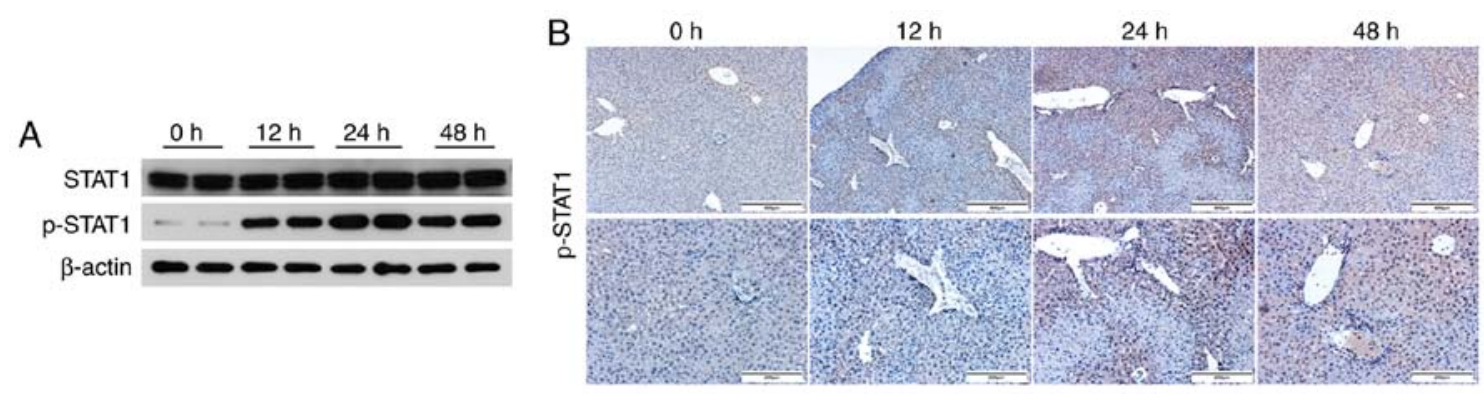

Figure 2. The JAK1/STAT1 pathway is activated in mice with ConA-induced hepatitis. (A) Western blot analyses of STAT1 and p-STAT1 expression in the liver tissue samples from the ConA-induced acute hepatitis mouse model at different time-points $(n=6)$. (B) The expression of p-STAT1 in the mouse livers was detected by immunohistochemical staining ( $\mathrm{n}=6 ; \mathrm{x} 100$ and x200 magnification). STAT1, signal transducers and activators of transcription 1; p-STAT1, phosphorylated STAT1.

A

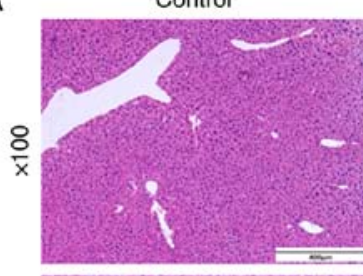

Tofacitinib
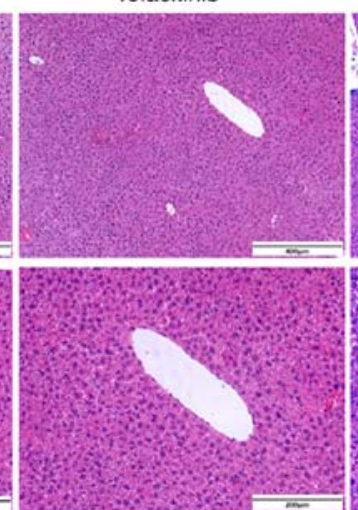

ConA
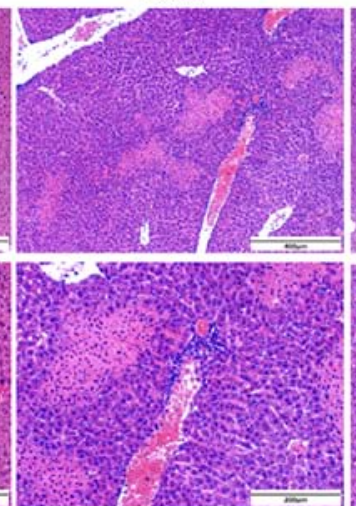

ConA+Tofacitinib
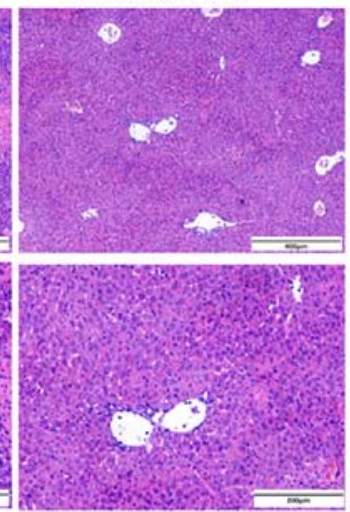

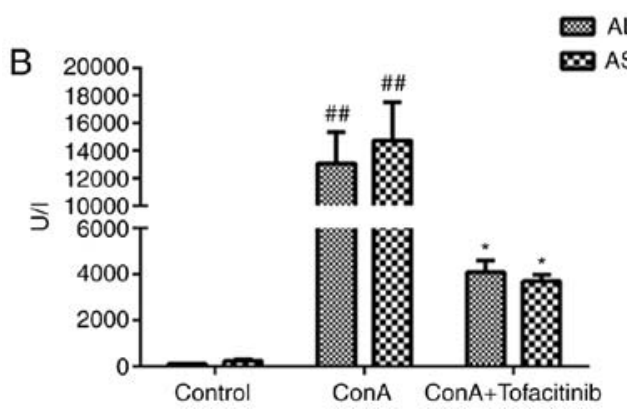

C
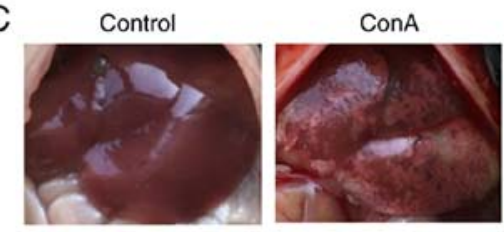

ConA+Tofacitinib

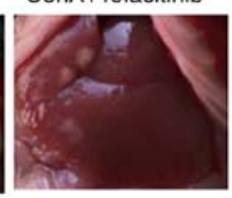

Figure 3. Tofacitinib effectively ameliorates inflammation in mice with ConA-induced acute hepatitis. (A) Male C57BL/6 mice that were administered with tofacitinib in advance by gavage were sacrificed $24 \mathrm{~h}$ after the ConA injection. Representative H\&E staining of the liver tissues was performed to determine the therapeutic effects of tofacitinib ( $\mathrm{n}=6$; x100 and x200 magnification). (B) The serum levels of ALT and AST were detected using an automatic biochemistry analysis apparatus $(\mathrm{n}=6) .{ }^{\# \#} \mathrm{P}<0.01$ vs. the control group. ${ }^{*} \mathrm{P}<0.05$ vs. the ConA-treated group. (C) Representative gross observation of the livers of the mice from the different groups that were administered with tofacitinib was performed $(n=6)$. ConA, concanavalin A; ALT, alanine transaminase; AST, aspartate transaminase.

whether its effects are dose-dependent. The mice in each group were sacrificed $24 \mathrm{~h}$ post-ConA injection. The mice injected with ConA developed hypothermia, becoming unresponsive and inactive. They curled up in the cage and were unusually quiet. However, the body temperature of the mice in the treatment group was much higher and these mice were in better condition. H\&E staining revealed that tofacitinib alleviated the histological liver damage (Fig. 3A) and significantly decreased the upregulated serum transaminase expression (Fig. 3B) in the ConA-injected mice. The gross appearance of the liver also provided strong evidence of the inflammation-relieving effect of tofacitinib from another perspective (Fig. 3C). Moreover, liver damage was alleviated to a greater extent in the mice administered with 10 or $15 \mathrm{mg} / \mathrm{kg} / \mathrm{day}$ of tofacitinib than in those administered with $5 \mathrm{mg} / \mathrm{kg} /$ day of tofacitinib (data not shown). However, there was no significant difference between the experimental groups comprised of mice administered with $10 \mathrm{mg} / \mathrm{kg} /$ day of tofacitinib and those administered with $15 \mathrm{mg} / \mathrm{kg} /$ day of tofacitinib; thus, only the results of the group comprised of the mice that were administered with $10 \mathrm{mg} / \mathrm{kg} /$ day of tofacitinib were presented.

Tofacitinib suppresses the expression of pro-inflammatory cytokines in mice with ConA-induced hepatitis. A mouse CBA kit was used to detect important cytokines (IL-2, IL-4, IL-6, IFN- $\gamma$, TNF- $\alpha$, IL-17A and IL-10) in the serum of mice from each experimental group; the data are presented in Fig. 4A. As observed in the results, the expression of some 


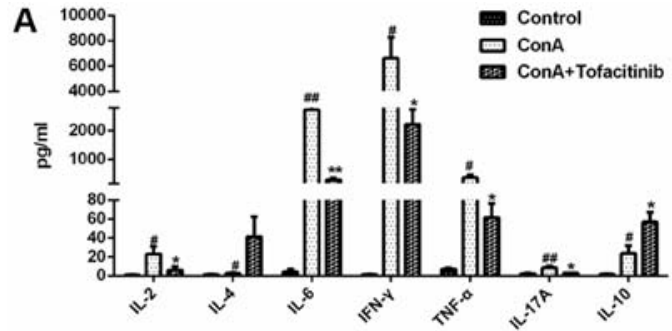

B
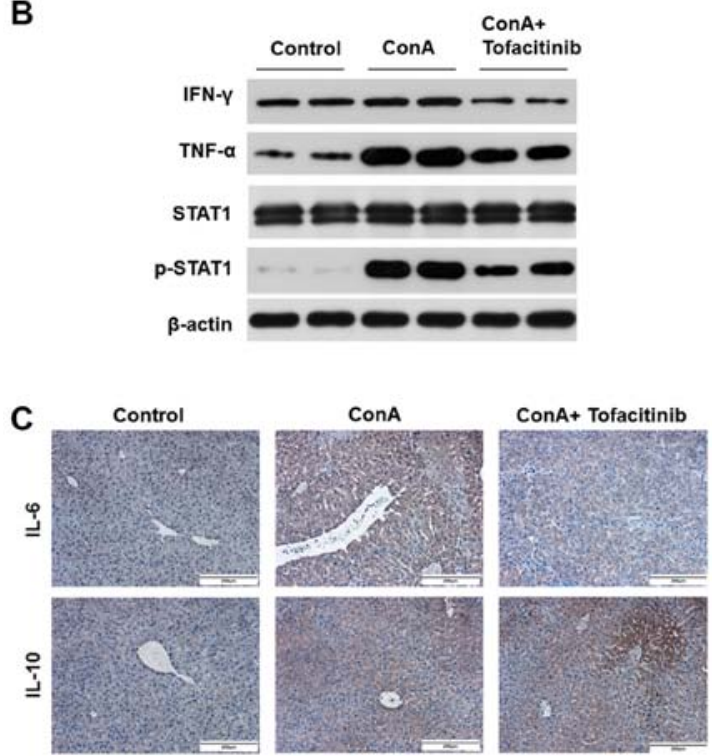

Figure 4. Tofacitinib suppresses the hepatic expression of pro-inflammatory cytokines in mice with ConA-induced hepatitis. (A) Several cytokines in the serum of mice from each group were detected using a CBA kit $(\mathrm{n}=3) .{ }^{\sharp} \mathrm{P}<0.05$, ${ }^{\# \#} \mathrm{P}<0.01$ vs. the control group. ${ }^{*} \mathrm{P}<0.05,{ }^{* *} \mathrm{P}<0.01$ vs. the ConA-treated group. (B) Western blotting was used to analyze the expression of IFN- $\gamma$ and TNF- $\alpha$ in the liver tissue samples from the mouse model with ConA-induced acute hepatitis $(n=6)$. (C) Immunohistochemical staining was performed to detect the expression of IL- 6 and IL-10 in the mouse livers ( $\mathrm{n}=6$; $x 200$ magnification). ConA, concanavalin A; ALT, alanine transaminase; AST, aspartate transaminase; TNF- $\alpha$, tumor necrosis factor- $\alpha$; IFN- $\gamma$, interferon- $\gamma$; IL-6, interleukin 6; IL-10, interleukin 10.

pro-inflammatory cytokines, such as IL-2, IL-6, IFN- $\gamma$, TNF- $\alpha$, and IL-17A, was upregulated after the ConA injection and revealed a downward trend in mice from the tofacitinib treatment group, while the expression of the anti-inflammatory cytokines, such as IL-4 and IL-10, increased after the tofacitinib treatment. The protein expression levels of TNF- $\alpha$ and IFN- $\gamma$ significantly increased in the whole liver extracts from mice in the ConA-treated groups, while the pretreatment with tofacitinib downregulated these expression levels (Fig. 4B). Considering that IL- 6 and IL-10 are the representative cytokines contributing to the distinct differentiation of $\mathrm{T}$ cells, immunohistochemical staining was further used to confirm the results presented in Fig. 4A (Fig. 4C). These results collectively confirmed that tofacitinib regulated the secretion level of anti- and pro-inflammatory cytokines under conditions of immune-mediated hepatitis.

Tofacitinib restores the impaired Treg/Th17 cell ratio under conditions of ConA-induced hepatitis. The imbalance of Tregs and Th17 cells plays a key role in the pathogenesis of many immune-mediated diseases; this imbalance may be regulated by different types of cytokines. ConA can induce severe inflammation in the mouse liver and cause the upregulation of pro-inflammatory cytokine expression in the serum. In addition, many autoimmune diseases are characterized by systemic disorders. Therefore, the ratio of Treg/Th17 cells was detected not only in the mouse liver, but also in the spleen, which is representative of the situation in the peripheral regions. Con A induced an increase in the number of Th17 cells in both the liver and spleen (Fig. 5A and C); the number of Tregs also increased. This increase occurred because Tregs, which are immunoregulatory cells, respond to the acute inflammation caused by ConA. Notably, the number of Tregs in mice from the treatment group exhibited an even greater increase, while the number of Th17 cells in the same group exhibited a downward trend. Although the number of both Tregs and Th17 cells exhibited an increasing tendency, the ratio of Treg/Th17 cells decreased following ConA injection (Fig. 5B and D). However, in the tofacitinib treatment group, the impaired Treg/Th17 cell ratio was significantly recovered.

Tofacitinib relieves liver fibrosis under conditions of AIH. Although the mouse model of ConA-induced immune-mediated liver injury resembles conditions of AIH in humans (38), chronic inflammation and liver fibrosis are not observed in this model. Evidence has revealed that the balance of Tregs and Th17 cells is involved in the liver fibrosis associated with chronic immune-mediated hepatitis $(39,40)$. Therefore, an AIH mouse model was used to further explore the effects of tofacitinib under conditions of liver fibrosis during immune-mediated hepatitis. Evident liver fibrosis appeared in the livers of mice with AIH and was in fact alleviated following tofacitinib treatment; representative Sirius red staining images are presented in Fig. 6. These data confirmed, from another point of view, that tofacitinib could suppress immune-mediated liver injury.

\section{Discussion}

Recently,anincreaseinthenumberof cases of immune-mediated liver injury, such as those involving autoimmune liver diseases, has been reported; this has attracted a great amount of attention from researchers (2). However, there is no effective therapy for these diseases apart from immunosuppressants (41). Moreover, the side effects of long-term standard immunosuppression and the lack of response to standard immunosuppressive therapy are the major clinical challenges associated with this treatment strategy (42). In recent years, some researchers have also considered whether certain interventions used to treat other autoimmune diseases such as rheumatic disorders, including treatment with CTLA-4 Ig, recombinant IL-10, and anti-TNF- $\alpha$ antibodies, and the adoptive transfer of Tregs, can be used for treating immune-mediated hepatitis (43). However, these alternative strategies have still not been applied widely in clinical settings.

Tofacitinib is an inhibitor of the members of the JAK family, which are intracellular proteins crucial to the downstream regulation of many inflammatory mediators (44). Tofacitinib is used to treat rheumatoid arthritis and is effective in methotrexate-naïve and DMARD-experienced patients, including those who are unresponsive to TNF inhibitors $(26,45,46)$. Recently, some studies revealed that tofacitinib also has a protective effect against other diseases caused by 


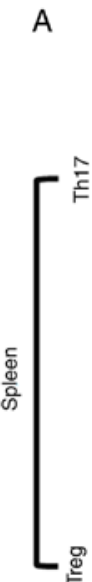
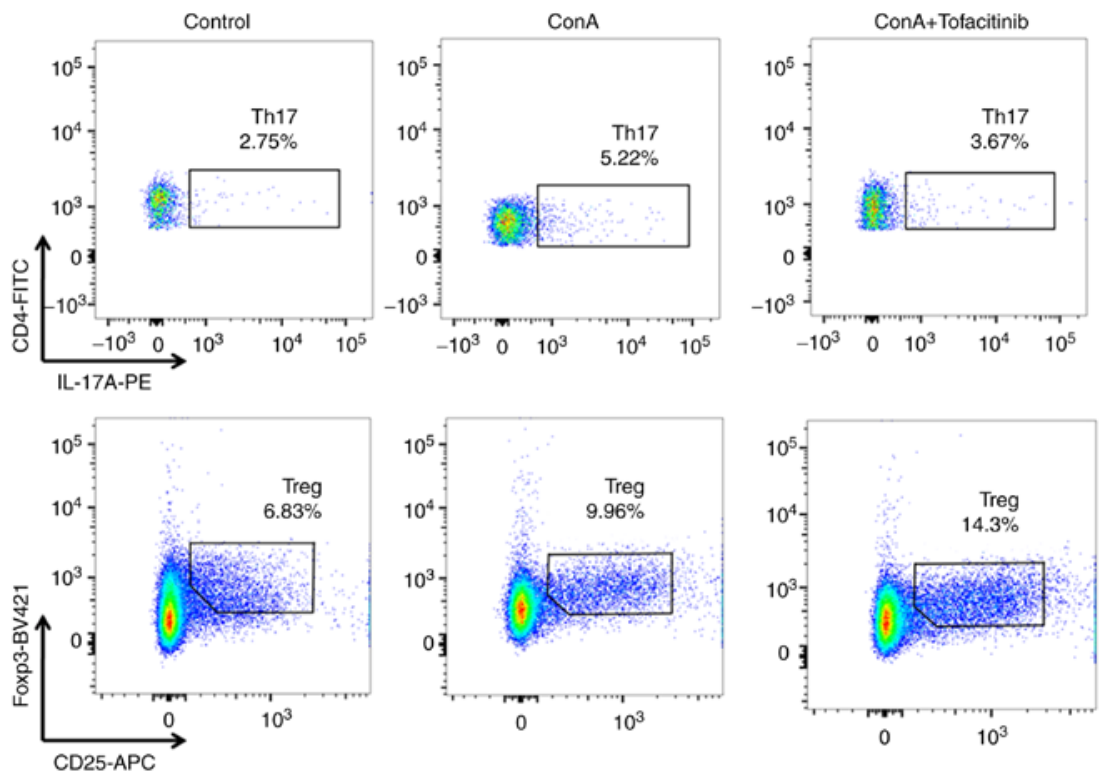

B

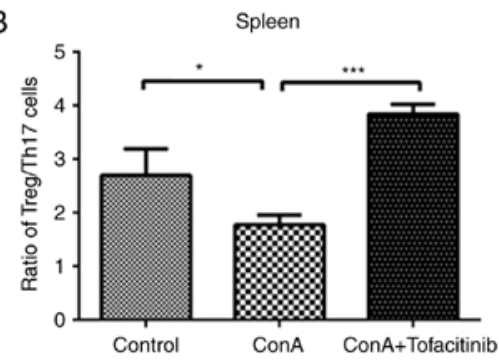

$\mathrm{C}$
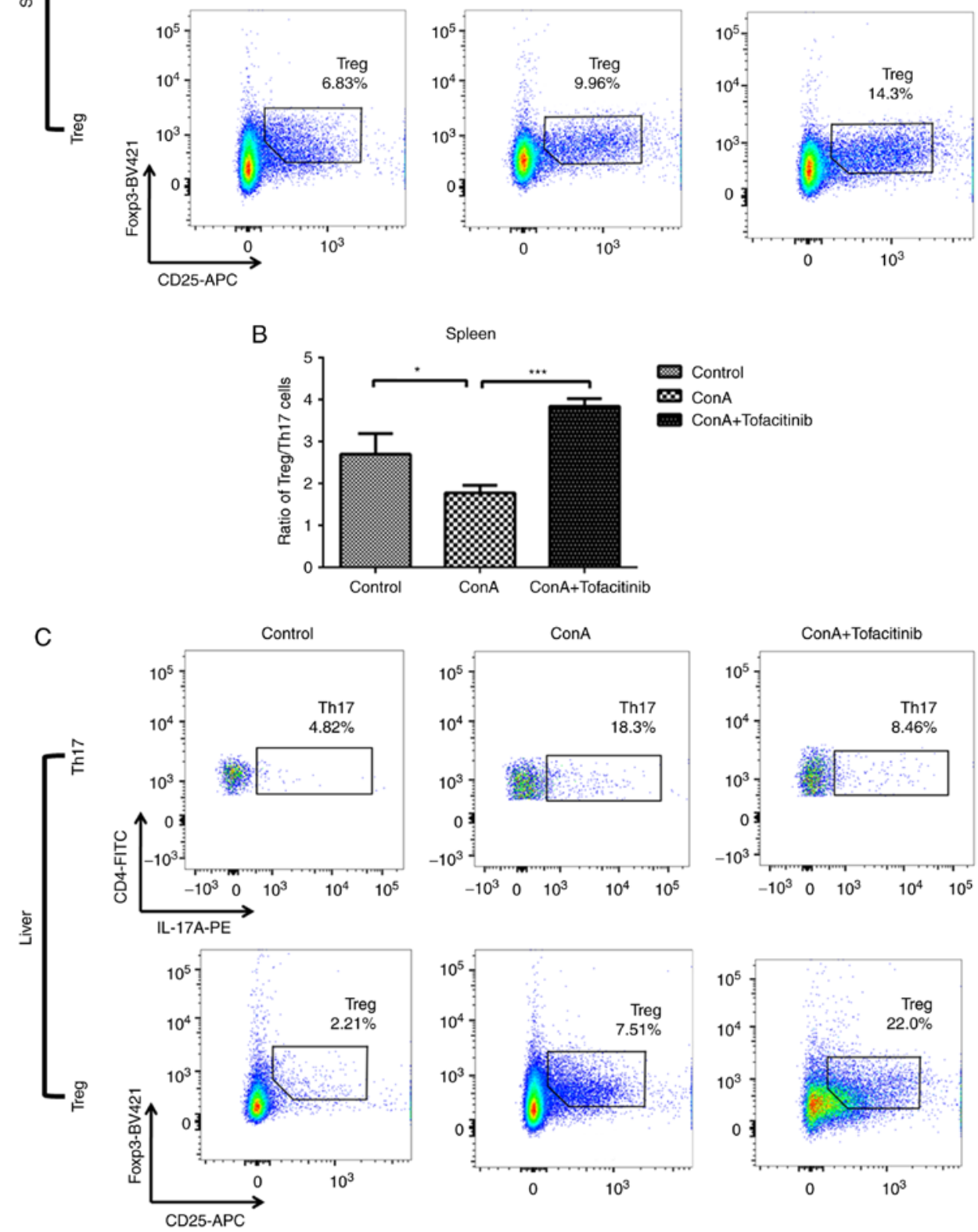

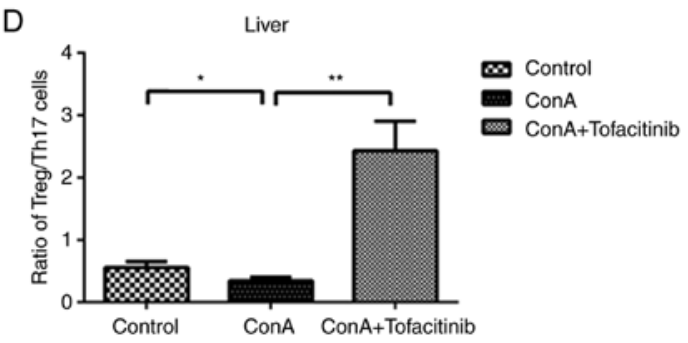

Figure 5. Tofacitinib restores the impaired Treg/Th17 cell ratio in mice with ConA-induced hepatitis. (A and C) Mononuclear cells were harvested from the livers $24 \mathrm{~h}$ after the ConA injection. The percentages of $\mathrm{CD} 4^{+} \mathrm{CD} 25^{+} \mathrm{Foxp} 3^{+}$Tregs and $\mathrm{CD} 4{ }^{+} \mathrm{IL}-17 \mathrm{~A}^{+}$Th17 cells were examined in the spleens and livers of the mice from the different experimental groups by flow cytometry $(\mathrm{n}=5$ ). Representative images are presented. (B and D) Statistical graph of the Treg/Th17 cell ratio in the livers and spleens of mice from the different experimental groups. ${ }^{*} \mathrm{P}<0.05,{ }^{* *} \mathrm{P}<0.01,{ }^{* * * *} \mathrm{P}<0.001$. ConA, concanavalin A; Treg, regulatory T cells; Th17, Th17 cells. 


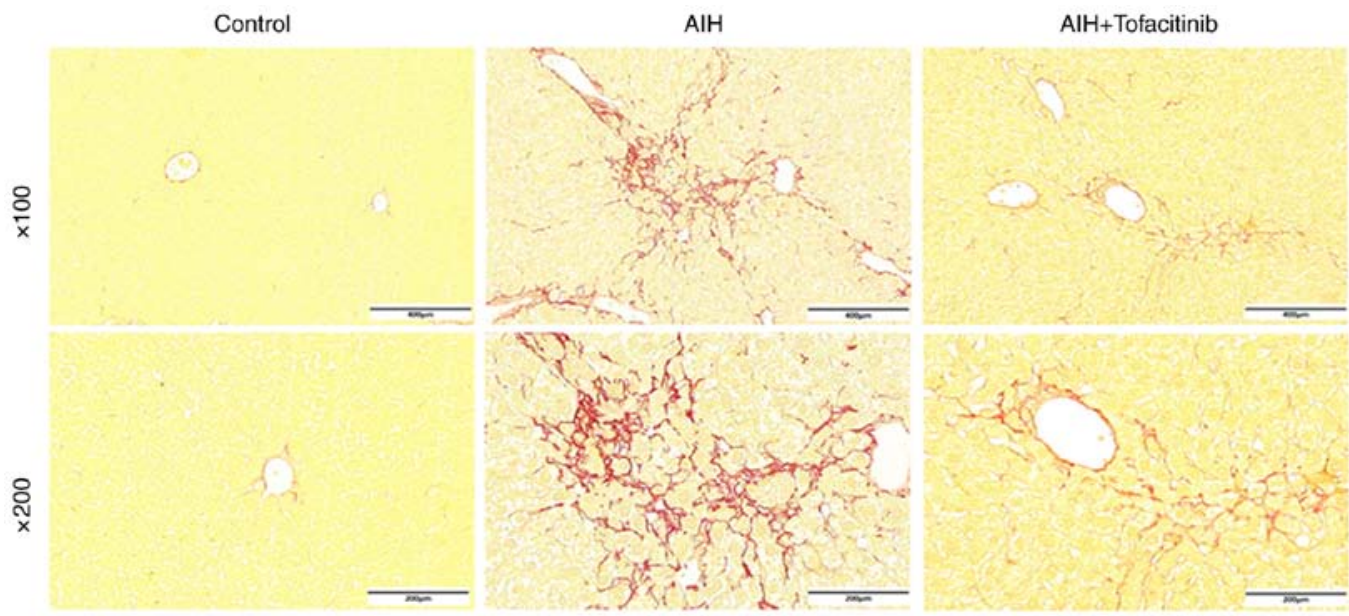

Figure 6. Tofacitinib relieves the liver fibrosis in mice with autoimmune hepatitis. Mice received an i.v. injection of adenovirus (Ad)/pCYP2D6 to induce AIH. Obvious liver fibrosis appeared on day 35 post the first i.v. injection. Mice with AIH were administered with tofacitinib intragastrically from day 35 onwards, and were sacrificed on day $49(\mathrm{n}=6)$. Liver fibrosis was detected by Sirius red staining and the representative pictures are presented. AIH, autoimmune hepatitis.

immune disorders, such as inflammatory bowel disease (47) and alopecia universalis $(48,49)$. It was observed that cytokines and the JAK/STAT pathways they participate in also play important roles in patients with AIH (50) and mice with ConA-induced hepatitis (51); this has led to the investigation of whether tofacitinib can also play a protective role against immune-mediated hepatitis. However, no studies related to this aspect have been reported. In the present study, to the best of our knowledge, for the first time, the protective effects of tofacitinib against immune-mediated liver injury were detected in a mouse model of ConA-induced immune-mediated hepatitis, which is a well-established animal model for the study of T cell-mediated hepatitis (14).

In the present study, a mouse model of ConA-induced immune-mediated hepatitis was first established and the JAK1/STAT1 pathway in the mouse livers was detected. It was revealed that the plasma transaminase levels and the expression levels of TNF- $\alpha$ and IFN- $\gamma$ peaked at $12 \mathrm{~h}$ after ConA injection, but the activation of the JAK1/STAT1 pathway appeared to occur later, perhaps because the release of the inflammatory cytokines that activate the JAK/STAT pathway proteins or the expression of JAK/STAT pathway proteins was delayed. It was revealed that the selective JAK inhibitor tofacitinib could in fact ameliorate mouse liver inflammation effectively. The CBA results revealed significant changes in the levels of several cytokines in the mouse serum. TNF- $\alpha$ and IFN- $\gamma$ are regarded as the most important mediators of ConA-induced hepatitis $(52,53)$, and their expression levels revealed a decreasing tendency in the mice from the tofacitinib treatment groups. The level of IL-6, which is essential for the differentiation of Th17 cells, was elevated after the ConA injection, but decreased following tofacitinib treatment; similar results were observed in case of IL-17A. Collectively, these results indicated that Th17 cells are involved in the protective effect of tofacitinib. However, the expression of IL-10, an anti-inflammatory cytokine related to tissue remodeling, Treg differentiation, and immune homeostasis, also increased after the ConA injection, perhaps due to a reaction elicited by the severe inflammation. Furthermore, IL-10 expression exhibited a further increase in mice from the tofacitinib treatment group, indicating that tofacitinib enhanced immune suppression in the mice.
Tregs and Th17 cells are the most important subtypes of $\mathrm{CD}^{+}$effector T cells associated with $\mathrm{AIH}$ and ConA-induced hepatitis. The balance between these cell types is the key to immune tolerance, and an impaired Treg/Th17 cell ratio participates in the pathogenesis of many autoimmune diseases. Considering that IL-10 is the key cytokine for Treg differentiation and IL- 6 induces the differentiation of naïve $\mathrm{CD} 4^{+} \mathrm{T}$ cells into Th17 cells, and that according to our results, the IL-10 and IL-6 levels were altered following tofacitinib treatment, MNCs were isolated from the livers of the experimental mice and the Treg/Th17 cell ratios were analyzed. Herein, it was first demonstrated that the number of both Tregs and Th17 cells increased during immune-mediated liver injury. However, the ratio of Tregs/Th17 cells decreased after the ConA injection and was notably restored in the tofacitinib treatment group, thereby strongly confirming our previous hypothesis. Tregs maintain immunological tolerance and prevent autoimmunity by inhibiting the activation and proliferation of effector $\mathrm{T}$ cells. In the mouse model of ConA-induced immune-mediated liver injury, Tregs played an important immunosuppressive role against severe liver inflammation and finally contributed to immune tolerance. The inflammation in the mouse liver was severe at $24 \mathrm{~h}$, and according to the present results, the number of both Th17 cells and Tregs increased. However, the ratio of Tregs/Th17 cells among $\mathrm{CD}^{+} \mathrm{T}$ cells decreased at $24 \mathrm{~h}$, suggesting that the number of Th17 cells increased more rapidly than that of Tregs at this time-point. However, the present results indicated that tofacitinib improved this situation and significantly restored the ratio of Treg/Th17 cells, resulting in the development of immune tolerance in the mouse livers in a much shorter time.

Although the mouse model of ConA-induced hepatitis is a well-recognized and typical tool to study T-cell-dependent liver injury, hepatitis in this model is acute and disappears after several days. Therefore, it was further confirmed that tofacitinib could suppress liver fibrosis in an AIH mouse model, which provides additional evidence for the protective effect of tofacitinib against immune-mediated liver injury. In summary, the aforementioned results indicated that tofacitinib can attenuate immune-mediated liver injury in mice, and this treatment effect was associated with the regulation of the Treg/Th17 
cell ratio and the inhibition of the expression of inflammatory cytokines. The present study indicated that tofacitinib is very likely to serve as a new treatment for immune-mediated liver diseases, providing a prospective therapeutic strategy for liver disease-related clinical challenges.

\section{Acknowledgements}

Not applicable.

\section{Funding}

The present study was supported by the National Natural Science Foundation of China (grant nos. 81600448, 81572419 and 81700515).

\section{Availability of data and materials}

The datasets used and/or analyzed are available from the corresponding author on reasonable request.

\section{Authors' contributions}

HW, YL and YX performed the experiments and contributed to the manuscript writing. XF and PH analyzed the data. DT and WY designed the experiments and contributed to data analysis. All authors read and approved the final manuscript and agree to be accountable for all aspects of the research in ensuring that the accuracy or integrity of any part of the work are appropriately investigated and resolved.

\section{Ethics approval and consent to participate}

The animal studies were approved by the Ethics Committee of Animal Experiments of Tongji Medical College (Wuhan, China) and monitored by the Department of Experimental Animals of Tongji Medical College (Wuhan, China).

\section{Patient consent for publication}

Not applicable.

\section{Competing interests}

The authors declare that they have no competing interests.

\section{References}

1. Eksteen B, Afford SC, Wigmore SJ, Holt AP and Adams DH: Immune-mediated liver injury. Semin Liver Dis 27: 351-366, 2007.

2. Webb GJ, Hirschfield GM, Krawitt EL and Gershwin ME: Cellular and molecular mechanisms of autoimmune hepatitis. Annu Rev Pathol 13: 247-292, 2018.

3. Manns MP, Czaja AJ, Gorham JD, Krawitt EL, Mieli-Vergani G, Vergani D and Vierling JM; American Association for the Study of Liver Diseases: Diagnosis and management of autoimmune hepatitis. Hepatology 51: 2193-2213, 2010.

4. Liberal R, Grant CR, Yuksel M, Graham J, Kalbasi A, Ma Y Heneghan MA, Mieli-Vergani G, Vergani D and Longhi MS: Regulatory $\mathrm{T}$-cell conditioning endows activated effector $\mathrm{T}$ cells with suppressor function in autoimmune hepatitis/autoimmune sclerosing cholangitis. Hepatology 66: 1570-1584, 2017.
5. Romano M, Fanelli G, Tan N, Nova-Lamperti E, McGregor R, Lechler RI, Lombardi G and Scottà C: Expanded regulatory $T$ cells induce alternatively activated monocytes with a reduced capacity to expand T helper-17 cells. Front Immunol 9: 1625, 2018.

6. Beringer A and Miossec P: IL-17 and IL-17-producing cells and liver diseases, with focus on autoimmune liver diseases. Autoimmun Rev 17: 1176-1185, 2018.

7. Longhi MS, Liberal R, Holder B, Robson SC, Ma Y, Mieli-Vergani $G$ and Vergani D: Inhibition of interleukin-17 promotes differentiation of $\mathrm{CD} 25^{-}$cells into stable $\mathrm{T}$ regulatory cells in patients with autoimmune hepatitis. Gastroenterology 142: 1526-1535.e6, 2012.

8. Sun XF, Gu L, Deng WS and Xu Q: Impaired balance of T helper $17 / \mathrm{T}$ regulatory cells in carbon tetrachloride-induced liver fibrosis in mice. World J Gastroenterol 20: 2062-2070, 2014.

9. Noack M and Miossec P: Th17 and regulatory T cell balance in autoimmune and inflammatory diseases. Autoimmun Rev 13: 668-677, 2014.

10. Tiegs G, Hentschel J and Wendel A: A T cell-dependent experimental liver injury in mice inducible by concanavalin A. J Clin Invest 90: 196-203, 1992.

11. Ji YR, Kim HJ, Bae KB, Lee S, Kim MO and Ryoo ZY: Hepatic serum amyloid A1 aggravates $\mathrm{T}$ cell-mediated hepatitis by inducing chemokines via Toll-like receptor 2 in mice. J Biol Chem 290: 12804-12811, 2015.

12. Erhardt A, Biburger M, Papadopoulos T and Tiegs G: IL-10, regulatory $T$ cells, and Kupffer cells mediate tolerance in concanavalin A-induced liver injury in mice. Hepatology 45: 475-485, 2007.

13. Shinohara $\mathrm{Y}$ and Tsukimoto $\mathrm{M}$ : Adenine nucleotides attenuate murine $\mathrm{T}$ cell activation induced by concanavalin A or $\mathrm{T}$ cell receptor stimulation. Front Pharmacol 8: 986, 2018.

14. Wang HX, Liu M, Weng SY, Li JJ, Xie C, He HL, Guan W, Yuan YS and Gao J: Immune mechanisms of Concanavalin A model of autoimmune hepatitis. World J Gastroenterol 18: 119-125, 2012.

15. Küsters S, Gantner F, Künstle G and Tiegs G: Interferon gamma plays a critical role in $\mathrm{T}$ cell-dependent liver injury in mice initiated by concanavalin A. Gastroenterology 111: 462-471, 1996.

16. Saitis A, Gatselis N, Zachou K and Dalekos GN: Use of TNF $\alpha$ antagonists in refractory AIH: Revealing the unforeseen. J Hepatol 59: 197-198, 2013.

17. Ehser J, Holdener M, Christen S, Bayer M, Pfeilschifter JM, Hintermann E, Bogdanos D and Christen U: Molecular mimicry rather than identity breaks T-cell tolerance in the CYP2D6 mouse model for human autoimmune hepatitis. J Autoimmun 42: 39-49, 2013.

18. Hintermann E, Ehser J, Bayer M, Pfeilschifter JM and Christen U: Mechanism of autoimmune hepatic fibrogenesis induced by an adenovirus encoding the human liver autoantigen cytochrome P450 2D6. J Autoimmun 44: 49-60, 2013.

19. Hardtke-Wolenski M, Dywicki J, Fischer K, Hapke M, Sievers M, Schlue J, Anderson MS, Taubert R, Noyan F, Manns MP and Jaeckel E: The influence of genetic predisposition and autoimmune hepatitis inducing antigens in disease development. J Autoimmun 78: 39-45, 2017.

20. Holdener M, Hintermann E, Bayer M, Rhode A, Rodrigo E, Hintereder G, Johnson EF, Gonzalez FJ, Pfeilschifter J, Manns MP, et al: Breaking tolerance to the natural human liver autoantigen cytochrome P450 2D6 by virus infection. J Exp Med 205: 1409-1422, 2008.

21. Christen U, Holdener M and Hintermann E: Cytochrome P450 2D6 as a model antigen. Dig Dis 28: 80-85, 2010.

22. Ghoreschi K, Laurence A and O'Shea JJ: Janus kinases in immune cell signaling. Immunol Rev 228: 273-287, 2009.

23. Shuai K and Liu B: Regulation of JAK-STAT signalling in the immune system. Nat Rev Immunol 3: 900-911, 2003.

24. Perner F, Schnoder TM, Ranjan S, Wolleschak D, Ebert C, Pils MC, Frey S, Polanetzki A, Fahldieck C, Schönborn U, et al: Specificity of JAK-kinase inhibition determines impact on human and murine T-cell function. Leukemia 30: 991-995, 2016.

25. Vainchenker W and Constantinescu SN: JAK/STAT signaling in hematological malignancies. Oncogene 32: 2601-2613, 2013.

26. Dhillon S: Tofacitinib: A review in rheumatoid arthritis. Drugs 77: 1987-2001, 2017.

27. Boyle DL, Soma K, Hodge J, Kavanaugh A, Mandel D, Mease P, Shurmur R, Singhal AK, Wei N, Rosengren S, et al: The JAK inhibitor tofacitinib suppresses synovial JAK1-STAT signalling in rheumatoid arthritis. Ann Rheum Dis 74: 1311-1316, 2015. 
28. Mease P, Hall S, FitzGerald O, van der Heijde D, Merola JF Avila-Zapata F, Cieślak D, Graham D, Wang C, Menon S, et al: Tofacitinib or Adalimumab versus placebo for psoriatic arthritis. N Engl J Med 377: 1537-1550, 2017.

29. Pouillon L, Bossuyt P and Peyrin-Biroulet L: Tofacitinib is the right OCTAVE for ulcerative colitis. Gastroenterology 153 862-864, 2017

30. Bayart CB, DeNiro KL, Brichta L, Craiglow BG and Sidbury R: Topical Janus kinase inhibitors for the treatment of pediatric alopecia areata. J Am Acad Dermatol 77: 167-170, 2017.

31. Ghoreschi K, Jesson MI, Li X, Lee JL, Ghosh S, Alsup JW, Warner JD, Tanaka M, Steward-Tharp SM, Gadina M, et al: Modulation of innate and adaptive immune responses by tofacitinib (CP-690,550). J Immunol 186: 4234-4243, 2011.

32. Nakamura $S$, Maehara $T$, Watanabe $S$, Ishihara $M$ and Sato $M$ Improvement of hydrodynamics-based gene transfer of nonviral DNA targeted to murine hepatocytes. Biomed Res Int 2013: 928790, 2013.

33. Vidal B, Cascao R, Finnila MA, Lopes IP, da Glória VG, Saarakkala S, Zioupos P, Canhão H and Fonseca JE: Effects of tofacitinib in early arthritis-induced bone loss in an adjuvant-induced arthritis rat model. Rheumatology (Oxford) 57: 1461-1471, 2018

34. Feng XX, Chi G, Wang H, Gao Y, Chen Q, Ru YX, Luo ZL, Yan W, Li PY, Liu M, et al: IL-37 suppresses the sustained hepatic IFN- $\gamma / \mathrm{TNF}-\alpha$ production and T cell-dependent liver injury. Int Immunopharmacol 69: 184-193, 2019.

35. Chi G, Feng XX, Ru YX, Xiong T, Gao Y, Wang H, Luo ZL, Mo R, Guo F, He YP, et al: TLR2/4 ligand-amplified liver inflammation promotes initiation of autoimmune hepatitis due to sustained IL-6/IL-12/IL-4/IL-25 expression. Mol Immunol 99: 171-181, 2018.

36. Livak KJ and Schmittgen TD: Analysis of relative gene expression data using real-time quantitative PCR and the 2(-Delta Delta C(T)) method. Methods 25: 402-408, 2001.

37. Chen X, Tan Q, Wang Y, Lv H, Wang Z, Lin Z, Du Z, Xiong S, Han J, Tian D and Wang B: Overexpression of KLF14 protects against immune-mediated hepatic injury in mice. Lab Invest 99: $37-47,2019$.

38. Ye T, Wang T, Yang X, Fan X, Wen M, Shen Y, Xi X, Men R and Yang L: Comparison of concanavalin a-induced murine autoimmune hepatitis models. Cell Physiol Biochem 46: 1241-1251, 2018.

39. Li J, Wang FP, She WM, Yang CQ, Li L, Tu CT, Wang JY and Jiang W: Enhanced high-mobility group box 1 (HMGB1) modulates regulatory T cells (Treg)/T helper 17 (Th17) balance via toll-like receptor (TLR)-4-interleukin (IL)-6 pathway in patients with chronic hepatitis B. J viral hepatitis 21: 129-140, 2014.

40. Li J, Qiu SJ, She WM, Wang FP, Gao H, Li L, Tu CT, Wang JY, Shen XZ and Jiang W: Significance of the balance between regulatory $\mathrm{T}$ (Treg) and $\mathrm{T}$ helper 17 (Th17) cells during hepatitis B virus related liver fibrosis. PLoS One 7: e39307, 2012.

41. Yang F, Wang Q, BianZ, Ren LL, Jia J and MaX: Autoimmune hepatitis: East meets west. J Gastroenterol Hepatol 30: 1230-1236, 2015.

42. Liberal R, Krawitt EL, Vierling JM, Manns MP, Mieli-Vergani G and Vergani D: Cutting edge issues in autoimmune hepatitis. J Autoimmun 75: 6-19, 2016.
43. Czaja AJ: Emerging opportunities for site-specific molecular and cellular interventions in autoimmune hepatitis. Dig Dis Sci 55: 2712-2726, 2010.

44. Lopez-Sanz L, Bernal S, Recio C, Lazaro I, Oguiza A, Melgar A, Jimenez-Castilla L, Egido J and Gomez-Guerrero C: SOCS1-targeted therapy ameliorates renal and vascular oxidative stress in diabetes via STAT1 and PI3K inhibition. Lab Invest 98: 1276-1290, 2018

45. Fleischmann R, Mysler E, Hall S, Kivitz AJ, Moots RJ, Luo Z, DeMasi R, Soma K, Zhang R, Takiya L, et al: Efficacy and safety of tofacitinib monotherapy, tofacitinib with methotrexate, and adalimumab with methotrexate in patients with rheumatoid arthritis (ORAL Strategy): A phase 3b/4, double-blind, head-to-head, randomised controlled trial. Lancet 390: 457-468, 2017.

46. Uttley L, Bermejo I, Ren S, Martyn-St James M, Wong R, Scott DL, Young A and Stevenson M: Tofacitinib for treating rheumatoid arthritis after the failure of disease-modifying anti-rheumatic drugs: An evidence review group perspective of a NICE single technology appraisal. Pharmacoeconomics 36: 1063-1072, 2018

47. White JR, Phillips F, Monaghan T, Fateen W, Samuel S, Ghosh S and Moran GW: Review article: Novel oral-targeted therapies in inflammatory bowel disease. Aliment Pharmacol Ther 47: 1610-1622, 2018 .

48. Patel NU, Oussedik E, Grammenos A and Pichardo-Geisinger R: A case report highlighting the effective treatment of alopecia universalis with tofacitinib in an adolescent and adult patient. J Cutan Med Surg 22: 439-442, 2018.

49. Silberstein SD, Yeung PP and Aycardi E: Preventive therapies for chronic migraine. N Engl J Med 378: 774, 2018.

50. Longhi MS, Hussain MJ, Mitry RR, Arora SK, Mieli-Vergani G, Vergani D and Ma Y: Functional study of CD4+CD25+ regulatory T cells in health and autoimmune hepatitis. J Immunol 176: 4484-4491, 2006.

51. Nakaya M, Hashimoto M, Nakagawa R, Wakabayashi $Y$, Ishizaki T, Takada I, Komai K, Yoshida $\mathrm{H}$ and Yoshimura A: SOCS3 in T and NKT cells negatively regulates cytokine production and ameliorates ConA-induced hepatitis. J Immunol 183: 7047-7053, 2009

52. Kato J, Okamoto T, Motoyama H, Uchiyama R, Kirchhofer D, Van Rooijen N, Enomoto H, Nishiguchi S, Kawada N, Fujimoto J and Tsutsui $\mathrm{H}$ : Interferon-gamma-mediated tissue factor expression contributes to T-cell-mediated hepatitis through induction of hypercoagulation in mice. Hepatology 57: 362-372, 2013.

53. Iwamoto S, Kido M, Aoki N, Nishiura H, Maruoka R, Ikeda A, Okazaki T, Chiba T and Watanabe N: TNF- $\alpha$ is essential in the induction of fatal autoimmune hepatitis in mice through upregulation of hepatic CCL20 expression. Clin Immunol 146: 15-25, 2013.

This work is licensed under a Creative Commons Attribution-NonCommercial-NoDerivatives 4.0 International (CC BY-NC-ND 4.0) License. 